\title{
Stability Analysis of SIQS Epidemic Model with Saturated Incidence Rate
}

\author{
O. Adebimpe ${ }^{1 *}$, L. M. Erinle-Ibrahim², A. F. Adebisi3 ${ }^{3}$ \\ ${ }^{1}$ Department of Physical Sciences, Landmark University, Omu-Aran, Nigeria \\ ${ }^{2}$ Department of Mathematics, Tai Solarin University of Education, Ijebu-Ode, Nigeria \\ ${ }^{3}$ Department of Mathematical and Physical Science, Osun State University, Osogbo, Nigeria \\ Email: *adebimpe.olukayode@lmu.edu.ng
}

Received 10 May 2015; accepted 29 July 2015; published 22 June 2016

Copyright (C) 2016 by authors and Scientific Research Publishing Inc.

This work is licensed under the Creative Commons Attribution International License (CC BY). http://creativecommons.org/licenses/by/4.0/

(c) (i) Open Access

\section{Abstract}

A SIQS epidemic model with saturated incidence rate is studied. Two equilibrium points exist for the system, disease-free and endemic equilibrium. The stability of the disease-free equilibrium and endemic equilibrium exists when the basic reproduction number $R_{0}$, is less or greater than unity respectively. The global stability of the disease-free and endemic equilibrium is proved using Lyapunov functions and Poincare-Bendixson theorem plus Dulac's criterion respectively.

\section{Keywords}

SIQS Epidemic Model, Saturated Incidence Rate, Basic Reproduction Number, Lyapunov Function, Poincare-Bendixson, Dulac Criterion

\section{Introduction}

The isolation and treatment of symptomatic individuals coupled with the quarantining of individuals that have a high risk of having been infected, constitute two commonly used epidemic control measures. Mass quarantine can inflict significant social, psychological and economic costs without resulting in the detection of many infected individuals. Day et al. [1], Hethcote et al. [2] considered SIQS and SIQR epidemic models with three forms of incidence, which include the bilinear, standard and quarantined-adjusted incidences.

Feng and Thieme [3] considered SEIQR models with arbitrarily distributed periods of infection, including quarantine and a general incidence assumed that all infected individuals go through the quarantine stage and investigated the model dynamics. Settapat and Wirawah [4] discussed the SIQ epidemic model with constant immigration. Yang et al. [5] also studied an SIQ epidemic model with isolation and nonlinear incidence rate.

\footnotetext{
${ }^{*}$ Corresponding author.
}

How to cite this paper: Adebimpe, O., Erinle-Ibrahim, L.M. and Adebisi, A.F. (2016) Stability Analysis of SIQS Epidemic Model with Saturated Incidence Rate. Applied Mathematics, 7, 1082-1086. 
El-Marouf and Alihaby [6] studied the equilibrium points and their local stability for SIQ and SIQR epidemic models with three forms of incidence rates. They also studied the global stability of the equilibrium by constructing the new forms of Lyapunov functions.

Gbadamosi and Adebimpe investigated an SIQ epidemic model with nonlinear incidence rate. They introduced the concept that describes the present and past states of the disease.

We extended the work of Gbadamosi and Adebimpe to include the rates at which individuals recover and return to susceptible compartment from compartments $I$ and $Q$ respectively and we apply Lyapunov functions and Poincare-Bendixson theorem plus Dulac's criterion to prove the global stability of disease-free and endemic equilibria respectively.

\section{The Model}

The model that governs a system of differential equation is presented as follows:

$$
\begin{aligned}
& \frac{\mathrm{d} S}{\mathrm{~d} t}=(1-p) A-\frac{\beta S I}{1+m I}-d S+\gamma I+\varepsilon Q \\
& \frac{\mathrm{d} I}{\mathrm{~d} t}=\frac{\beta S I}{1+m I}+p A-(\gamma+\delta+d+\alpha) I \\
& \frac{\mathrm{d} Q}{\mathrm{~d} t}=\delta I-(\varepsilon+d+\alpha) Q
\end{aligned}
$$

Subject to initial conditions

$$
S(0)=S_{0} \geq 0, \quad I(0)=I_{0} \geq 0, \quad Q(0)=Q_{0} \geq 0
$$

The parameters with their descriptions are presented in Table 1.

The addition of the system (1), gives

$$
\frac{\mathrm{d} N}{\mathrm{~d} t}=A-d N-\alpha I-\alpha Q \quad \text { where } N=S+I+Q
$$

From above equation, we get

$$
\begin{aligned}
& 0 \leq \lim _{t \rightarrow \infty} \sup N(t) \leq N_{0} \\
& \text { with } \lim _{t \rightarrow \infty} \sup N(t)=N_{0} \text { if and only if } \lim _{t \rightarrow \infty} \sup I(t)=0
\end{aligned}
$$

From the first equation of the system (1), it follows

$$
0 \leq \lim _{t \rightarrow \infty} \sup S(t) \leq S_{0}
$$

And the second equation gives

Table 1. Descriptions of parameters.

\begin{tabular}{ll}
\hline Parameter & Description \\
\hline$(1-p) A$ & Recruitment of the susceptible corresponding to Births and Immigration \\
$p A$ & constant recruitment of the infected compartment \\
$D$ & Per capita natural mortality rate \\
$\delta$ & The rate constant for individuals leaving the compartment $I$ for the quarantine compartment $Q$ \\
$\alpha$ & Disease-related death rate constant in compartments $I$ and $Q$ \\
$\gamma$ & Rate at which individuals recover and return to Susceptible (S) from compartment $I$ \\
$\varepsilon$ & Rate at which individuals recover and return to Susceptible (S) from compartment $Q$ \\
$M$ & The saturation constant \\
\hline
\end{tabular}




$$
0 \leq \lim _{t \rightarrow \infty} \sup I(t) \leq I_{0} .
$$

So, from the above, if $N>N_{0}$, then $\frac{\mathrm{d} N}{\mathrm{~d} t}<0$.

We can now write

$$
\Omega=\left\{(S, I, Q) \in R_{+}^{3}: S+I+Q \leq N_{0}, S \leq S_{0}, I \leq I_{0}\right\}
$$

\section{Equilibria}

The system (1) has always the disease-free equilibrium at $E_{0}=\left(S_{0}, I_{0}, Q_{0}\right)=\left(\frac{(1-p) A}{d}, 0,0\right)$

Endemic Equilibrium: $E_{*}=\left(S_{*}, I_{*}, Q_{*}\right)$

\section{Local Stability}

In this section, we discussed the local stability of the disease-free equilibrium and endemic equilibrium for the system (1).

We state and prove the following results:

Theorem 1: At $E_{0}$, the disease-free equilibrium of the system (1) is locally asymptotically stable when $R_{0}<1$.

Proof: The Jacobian matrix at the point $E_{0}$ through linearization is given by

$$
J_{0}=\left(\begin{array}{ccc}
-d & -\left(\beta S_{0}-\gamma\right) & \varepsilon \\
0 & \beta S_{0}-(\gamma+\delta+d+\alpha) & 0 \\
0 & \delta & -(\varepsilon+d+\alpha)
\end{array}\right)
$$

By finding the eigenvalues, we have the following $\lambda s$ :

$$
\lambda_{1}=-d, \quad \lambda_{2}=\beta S_{0}-(\gamma+\delta+d+\alpha), \quad \lambda_{3}=-(\varepsilon+d+\alpha)
$$

For $\lambda_{2}$ to be negative $\beta S_{0}<(\gamma+\delta+d+\alpha)$

That is, $\frac{\beta S_{0}}{\gamma+\delta+d+\alpha}<1$

Let $R_{0}=\frac{\beta(1-p) A}{d(\gamma+\delta+d+\alpha)}$

If $R_{0}=\frac{\beta(1-p) A}{d(\gamma+\delta+d+\alpha)}<1, \lambda_{2}<0$

Since $\lambda_{1}<0, \lambda_{3}<0$ and $\lambda_{2}<0$ if $R_{0}<1$, the disease-free equilibrium is locally asymptotically stable.

Theorem 3.1: The system (1) is locally asymptotically stable at $E_{*}$ if $R_{0}>1$, otherwise unstable.

Proof: At the endemic equilibrium $E_{*}$, the Jacobian matrix of the system (1) is given by:

$$
J_{*}=\left(\begin{array}{ccc}
-\left(\beta I_{*}+d\right) & -\beta S_{0}+\gamma & \varepsilon \\
\beta I_{*} & \beta S_{*}-(\gamma+\delta+d+\alpha) & 0 \\
0 & \delta & -(\varepsilon+d+\alpha)
\end{array}\right)
$$

The characteristic equation of the Jacobian matrix $J_{*}$ is given by

$$
\lambda^{3}+a_{1} \lambda^{2}+a_{2} \lambda+a_{3}
$$

where $a_{1}=3 \alpha+2 \delta+\gamma+3 d+\beta I_{*}+\varepsilon-2 \beta S_{*}$

$$
\begin{aligned}
a_{2}= & 2 \beta I_{*} \alpha+\beta I_{*} d+\beta I_{*} \delta+3 d^{2}+4 \alpha d+\alpha^{2}+\gamma \alpha+\delta \alpha+2 \delta d+2 \gamma d \\
& +3 d \varepsilon+\gamma \delta+\beta I_{*} \varepsilon+\beta I_{*} d-\beta S_{*} d-2 \beta S_{*} \varepsilon
\end{aligned}
$$




$$
\begin{aligned}
a_{3}= & \beta I_{*} d \varepsilon+\beta I_{*} \alpha \varepsilon+\gamma d \varepsilon+\delta d \varepsilon+d^{2} \varepsilon+\alpha d \varepsilon+\beta I_{*} \delta d+\beta I_{*} \alpha d+\gamma d^{2}+d^{3}+2 \alpha d^{2} \\
& +\beta I_{*} \alpha \delta+\beta I_{*} d \alpha+\beta I_{*} \alpha^{2}+\gamma \alpha d+\delta \alpha d+\alpha^{2} d-\beta S_{*} \alpha d
\end{aligned}
$$

as $a_{1}>0, a_{2}>0, a_{3}>0$ and $a_{1} a_{2}-a_{3}>0$

If $a_{1} a_{2}>a_{3}$, by Routh Hurwitz criterion, all the eigenvalues of the system (1) has negative real part. Therefore, the endemic equilibrium of the system (1) at $E_{*}$ is locally asymptotically stable.

\section{Global Stability}

In this section, we study the global stability of the disease-free equilibrium and endemic equilibrium by Lyapunov function and Poincare-Bendixson theorem respectively.

Theorem 3: (Dulac's Criterion)

Consider the following general nonlinear autonomous system of de

$$
x(t)=f(x), x \in E
$$

Let $f=C^{1}(E)$ where $E$ is a simple connected region in $R^{2}$. If the exists a function it $H \in C^{1}(E)$ such that $\nabla \cdot(H f)$ is not identically zero and does not change sign in $E$, the system $(*)$ has no close orbit lying entirely in $E$. if $\mathrm{A}$ is an annular region contained in $E$ on which $\nabla \cdot(H f)$ does not change sign, then there is at most one limit cycle of the system $(*)$ in A.

Theorem 4: (The Poincare-Bendixson Theorem):Suppose that $f \in C^{1}(E)$ where $E$ is an open subset of $R^{n}$ and that the system $\left({ }^{*}\right)$ has a rejecting $\Gamma$ contained in a compact subset $f$ of $E$. assume that the system (*) has only one unique equilibrium point $x_{0}$ in $f$, then one of the following possibilities holds.

(a) $w(\Gamma)$ is the equilibrium point $x_{0}$

(b) $w(\Gamma)$ is a periodic orbit

(c) $w(\Gamma)$ is a graphic

Theorem 5: The disease-free equilibrium of the model (1) is globally asymptotically stable if $R_{0}<1$

Proof: To prove this result, we construct the following Lyapunov function

$$
L=u_{1}\left(S-S_{0}\right)+u_{2}\left(I-I_{0}\right)+u_{3} Q
$$

where $u_{1}, u_{2}$ and $u_{3}$ are positive constants to be determined later. Differentiating equation (3) with respect to $t$, we obtain

$$
\begin{aligned}
L^{\prime}= & u_{1}\left[(1-p) A-\frac{\beta S I}{1+m I}-d S+\gamma I+\varepsilon Q\right]+u_{2}\left[\frac{\beta S I}{1+m I}+p A-(\gamma+\delta+d+\alpha) I\right] \\
& +u_{3}[\delta I-(\varepsilon+d+\alpha) Q]
\end{aligned}
$$

After rearrangements, we get

$$
\begin{aligned}
L^{\prime}= & \frac{\beta S I}{1+m I}\left(u_{2}-u_{1}\right)+p A\left(u_{2}-u_{1}\right)+\gamma I\left(u_{2}-u_{1}\right)+\varepsilon Q\left(u_{3}-u_{1}\right)+\delta I\left(u_{3}-u_{1}\right) \\
& +u_{1} A-u_{1} d S-u_{2} d I-u_{3} d Q-u_{2} \alpha I-u_{3} \alpha Q
\end{aligned}
$$

Let us choose the constants $u_{1}=u_{2}=u_{3}=1$. Finally, we obtain

$$
\begin{aligned}
& L^{\prime}=A-d(S+I+Q)-\alpha(I+Q) \\
& L^{\prime}=-(d N-A)-\alpha(N-S)<0
\end{aligned}
$$

Thus, the disease-free equilibrium of the system (1) is globally asymptotically stable if $R_{0}<1$ In the next theorem, we present the global stability of the endemic equilibrium of the system (1) at $E_{*}$ Theorem 6: The endemic equilibrium $E_{*}$ of the system (1) is globally asymptotically stable if $R_{0}>1$. Proof: In order to prove the result, we use Dulac plus Poincare Bendixson theorem as follow

$$
H(S, I, Q)=\frac{1}{S I Q} \text { where } S>0, I>0, Q>0 .
$$

Then, 


$$
\begin{aligned}
\nabla \cdot(H F)= & \frac{\partial}{\partial S}\left(H \cdot F_{1}\right)+\frac{\partial}{\partial I}\left(H \cdot F_{2}\right)+\frac{\partial}{\partial Q}\left(H \cdot F_{3}\right) \\
= & \frac{\partial}{\partial S}\left[\frac{1}{S I Q}\left((1-p) A-\frac{\beta S I}{1+m I}-d S+\gamma I+\varepsilon Q\right)\right]+\frac{\partial}{\partial I}\left[\frac{\beta S I}{1+m I}+p A-(\gamma+\delta+d+\alpha) I\right] \\
& +\frac{\partial}{\partial Q}[\delta I-(\varepsilon+d+\alpha) Q] \\
\nabla \cdot(H F)= & -\frac{(1-p) A}{S^{2} I Q}-\frac{\gamma}{S^{2} Q}-\frac{\varepsilon}{S^{2} I}-\frac{p A}{S I^{2} Q}-\frac{\delta}{S Q^{2}}<0
\end{aligned}
$$

Hence, by Dulac's criterion, there is no closed orbit in the first quadrant. Therefore, the endemic equilibrium is globally asymptotically stable.

\section{Discussion of Results}

The mathematical and stability analysis of SIQS epidemic model with saturated incidence rate and temporary immunity has been presented. We investigated the local stability of the disease-free equilibrium and endemic equilibrium using the basic reproduction number, $R_{0}$. We observed that, when $R_{0}<1$, the disease-free equilibrium is stable at $E_{0}$ locally and endemic equilibrium is unstable which means there is tendency for the disease to die out in the long run. We proved the global stability of the disease free equilibrium and endemic equilibrium of the model using Lyapunov function and Dulac's criterion plus Poincare-Bendixson theorem respectively.

\section{References}

[1] Day, T., Park, A., Madras, N., Gumel, A. and Wu, J. (2006) When Is Quarantine a Useful Control Strategy for Emerging Infectious Diseases? American Journal of Epidemiology, 163, 479-485. http://dx.doi.org/10.1093/aje/kwj056

[2] Hethcote, H., Ma, Z. and Liao, S. (2002) Effects of Quarantine in Six Endemic Models for Infectious Diseases. Mathematical Biosciences, 180, 141-160. http://dx.doi.org/10.1016/S0025-5564(02)00111-6

[3] Feng, Z. and Thieme, H.R. (2000) Endemic Models with Arbitrarily Distributed Periods of Infection, I. General Theory. SIAM Journal on Applied Mathematics, 61, 803-833. http://dx.doi.org/10.1137/S0036139998347834

[4] Settapat, C. and Wirawan, C. (2007) Global Stability of an SIQ Epidemic Model. Kasetsart Journal: Natural Science, 41, 225-228.

[5] Yang, X., Li, F. and Cheng, Y. (2012) Global Stability Analysis on the Dynamics of an SIQ Model with Nonlinear Incidence Rate. In: Advances in Future Computer and Control Systems, 2, Volume 160 of the series Advances in Intelligent and Soft Computing, 561-565.

[6] El-marouf, S.A.A. and Alihaby, S.M. (2011) Global Analysis of an Epidemic Model with Nonlinear Incidence Rate. Journal of Mathematics and Statistics, 7, 319-325. http://dx.doi.org/10.3844/jmssp.2011.319.325

\section{Submit or recommend next manuscript to SCIRP and we will provide best service for you:}

Accepting pre-submission inquiries through Email, Facebook, Linkedin, Twitter, etc

A wide selection of journals (inclusive of 9 subjects, more than 200 journals)

Providing a 24-hour high-quality service

User-friendly online submission system

Fair and swift peer-review system

Efficient typesetting and proofreading procedure

Display of the result of downloads and visits, as well as the number of cited articles

Maximum dissemination of your research work

Submit your manuscript at: http://papersubmission.scirp.org/ 